\title{
Review on Modeling and Control of Flexible Link Manipulators
}

\author{
Dipendra Subedi*, Ilya Tyapin and Geir Hovland \\ Department of Engineering Sciences, University of Agder, 4879 Grimstad, Norway. E-mail: *dipendra.subedi@uia.no
}

\begin{abstract}
This paper presents a review of dynamic modeling techniques and various control schemes to control flexible link manipulators (FLMs) that were studied in recent literature. The advantages and complexities associated with the FLMs are discussed briefly. A survey of the reported studies is carried out based on the method used for modeling link flexibility and obtaining equations of motion of the FLMs. The control techniques are reviewed by classifying them into two main categories: model-based and model-free control schemes. The merits and limitations of different modeling and control methods are highlighted.
\end{abstract}

Keywords: Flexible link manipulator, dynamic modeling, vibrations, control, elasticity, flexibility, deflection.

\section{Introduction}

Link flexibility is present in manipulators because of two main reasons: the use of lightweight material and the long or slender design. It introduces static and dynamic deflections of the link and end-effector. Neglecting link flexibility in modeling and control of robots causes static and dynamic errors while carrying out any task. The static error includes a steady-state error, and dynamic errors include vibration and tracking error. These errors result in the degradation of the overall performance of the FLMs. From the control point of view, mechanical flexibility introduces an additional problem of non-colocation between the input commands and typical outputs to be controlled. So, it is crucial to consider link flexibility during modeling and control design of FLMs.

Major advantages of FLMs over rigid, bulky robots (designed with highly stiff materials) include, but are not limited to, low cost, smaller actuators, reduced energy consumption, high payload-to-robot-weight ratio, high operational speed, better transportability, and safer operation (Kiang et al., 2015). Because of these underlying advantages, the research interest in FLMs has increased significantly in recent years. FLMs can be used in many engineering applications for performing different robotic operations such as space research (Sabatini et al., 2012; Jing et al., 2019; Comi et al., 2019), construction automation (Cao and Liu, 2020), offshore applications (wind farm, sea farm, fish farm, autonomous mooring), aerospace industry, robotic surgery (Li et al., 2015; Runciman et al., 2019), and applications that require physical interaction (Suarez et al., 2018) and human-robot collaboration.

In contrast to the rigid industrial robot, it is not possible to control the precise position and orientation of the end-effector of the FLMs to perform manipulation tasks using only the rigid body kinematic information and joint encoder readings. This is because link flexibility causes deflection of the links and unwanted oscillations, which causes problems in the precise position control of the end-effector and may even lead to an unstable system. To control the vibration of the FLMs, additional sensors like vision sensors, IMUs, are usually added to the FLMs control architecture to provide vibration measurement of the flexible links or the endeffector. 
For achieving minimum oscillations and good position accuracy, the industrial robots are designed with highly stiff materials (like heavy steel with bulky design), which consequently require expensive high-power drives. However, the vibration of the end-effector at high speed and high load is still present due to industrial robot joint elasticity. In this context, lightweight flexible manipulators are better (cheaper) alternatives if the control architecture is designed to reduce the vibration of the end-effector to an acceptable range (depending on the application).

The nonlinear dynamics of the system with an infinite number of degrees of freedom make control of FLMs more complicated than the conventional industrial robots. In order to develop an efficient modelbased control algorithms for the FLMs, it is necessary to construct a mathematical model of the system incorporating flexibility of the links. On the one hand, it is impractical to model the flexible link with infinite degrees of freedom for dynamic analysis and simulations, but on the other hand, it is challenging to describe the system with a finite degree of freedom and still being able to represent all the dynamically relevant flexibility effects. Nevertheless, the actual system behavior should be represented by a dynamically accurate and computationally affordable mathematical model to design a suitable (model-based) control algorithm. When designing an FLM, it is important to obtain dynamic features that avoid complex vibration modes, which will make it difficult to control the FLM. Therefore, modeling is essential for the model-based design of FLMs for optimal control performance. In general, additional generalized coordinates are introduced to describe flexibility in addition to the coordinates that are used to describe the rigid motion of the manipulator in a Lagrangian formulation. Because of the additional generalized coordinates, the FLMs have fewer actuators than the number of degrees of freedom. As a result of this type of under-actuation, it is more difficult to design suitable laws to control FLMs than to control rigid arm manipulator. Lochan et al. (2016a) described additional complexities involved in the flexible manipulator, including the problem of controller/observer spillover due to model truncation.

The study of modeling, control, and sensor systems for the application of FLMs are of on-going interest for researchers worldwide. With the recent advancement in technology and the emergence of newer robot applications, researchers are focusing on the accurate dynamic modeling of the FLMs, controlling and applying them into different applications.

Because of a huge number of research publications in FLMs, it is interesting to present an exhaustive review of different modeling techniques, dynamic models, control problems, control strategies, and complexities involved in FLMs that are studied by many researchers worldwide. Previously, the studies on FLMs were surveyed by Jing et al. (2019), Sayahkarajy et al. (2016), Alandoli et al. (2016), Lochan et al. (2016a), Kiang et al. (2015), Hussein (2015), Rahimi and Nazemizadeh (2014), Dwivedy and Eberhard (2006), and Benosman and Le Vey (2004).

Jing et al. (2019) reviewed different dynamic stable control methods along with kinematic analysis of space flexible manipulators. The control strategies reviewed in the paper included linear feedback control, nonlinear control, and adaptive control. Furthermore, they pointed out several problems with modeling of dynamics and stable control of FLMs and presented some suggestions for the stable dynamic control. Sayahkarajy et al. (2016) and Lochan et al. (2016a) surveyed different modeling methods and control schemes used for the two-link flexible manipulator. Alandoli et al. (2016) presented a review of different mathematical modeling and control techniques for FLMs by grouping them based on the number of links: single-link, two-link, and multi-link manipulators. Kiang et al. (2015) reviewed different modeling methods, control techniques, sensors used for FLMs, and flexible joint manipulators. Hussein (2015) focused the survey on different vision-based control approaches for FLMs. Rahimi and Nazemizadeh (2014) reported a literature survey on three commonly used dynamic modeling methods, namely lumped parameter, assumed model, and finite element methods, and three main intelligent control techniques, namely fuzzy logic, neural network, and genetic algorithm. Dwivedy and Eberhard (2006) reviewed the literature related to dynamic analysis of flexible joint/link manipulators by classifying them based on modeling, control, and experimental studies. They further categorized papers based on the number of links (single-link, two-link, and multilink manipulators) and method of analysis used in the studies (lumped parameter, assumed modes, finite element, and other methods). Benosman and Le Vey (2004) surveyed different control strategies for one-link, two-link, and multi-link flexible manipulators based on the studies published until 2004.

Besides a lot of research done in the field of modeling and control of FLMs in recent years (from 2016 to present), a rigorous review of different modeling and control techniques is not available to report the current state of the art. This paper provides an extensive review of different modeling and control techniques for FLMs available in literature up to 2020 and hence complements the earlier literature reviews. Besides, the merits and demerits of different modeling and control techniques are highlighted. 
The paper is organized into four sections as follows. The various techniques for modeling FLMs, along with their advantages and disadvantages, are summarized in section 2. A comprehensive review of different methods to control FLMs is presented in section 3 by categorizing them into model-based and model-free techniques. Conclusions and discussions follow in section 4, along with the suggestions on possible future directions for research on FLMs.

\section{Modeling of FLMs}

Some applications require the design of long and slender mechanical structures with infinite rigidity, which is always an ideal assumption. Such structures possess some degree of in-built flexibility, which is unavoidable because of the material used and length of the link. Moreover, in recent years, the use of lighter arms and cheaper gears by robot manufacturers is justifiable in order to compete with lower prices. In this context, it is necessary to incorporate the flexibility for accurate dynamic modeling of the system.

Apart from the complexities associated with modeling link flexibility with infinite degrees of freedom, there are other issues that are in common to rigid manipulators that need to be handled. One of them is Coulomb friction in actuators, gears, and other transmissions. Additionally, joint flexibility, non-linearities in gears as lost motion and backlash have to be included in the dynamic model of the FLM. This section emphasizes the research in modeling flexibility in the links of FLMs rather than general modeling issues in manipulators. A review of different methods commonly used to model flexible bodies and techniques to obtain equations of motion is presented.

\subsection{Methods of dynamic modeling of flexible bodies}

Different models of flexible bodies are available in the literature depending upon the assumptions and required complexity. The accuracy of the models depends on the assumptions made to simplify the complexity of the flexible link manipulator system. For example, the Euler-Bernoulli equation is often used to include bending of a flexible link that neglects the effects of shear deformation and rotary inertia, which results in an error in deflection estimation for highfrequency vibrations (Abe, 2009). There are four main approaches that are commonly used in the literature: lumped parameter method (LPM), assumed modes method (AMM), finite element method (FEM), and transfer matrix method (TMM). Apart from these common methods, there are other methods used for the development of the dynamic model of the FLMs which include, but are not limited to, perturbation method (Khadem and Pirmohammadi, 2003; Jonker and Aarts, 2001), pseudo-rigid body method (Yanan et al., 2017), global mode method (Wei et al., 2017), and modal integration method (Aarts and Jonker, 2002).

\subsubsection{Lumped parameter method (LPM)}

In this method, the flexible link is modeled as a set of mass, spring, and damper connected by a torsion spring joint (Subedi et al., 2020). Kim and Uchiyama (2003) used this method to model FLM to clarify the vibration mechanism of a constrained, multi-DOF, flexible manipulator, and to devise the suppression method.

Recently, researchers used the LPM to model flexible arms of multi-link manipulators (Giorgio and Del Vescovo, 2018), the boom of a mobile concrete pump (Wanner and Sawodny, 2019), and other flexible mechanical structures (Pappalardo and Guida, 2018). Lochan et al. (2016b) utilized LPM to model the dynamics of a two-link flexible manipulator. Sun et al. (2016), Cambera and Feliu-Batlle (2017), He et al. (2017a), and Cambera and Feliu-Batle (2018) modeled a single-link flexible manipulator system based on LPM. Pucher et al. (2019, 2020) described the elastic deflection of a 3-DOF robot with flexible links using LPM.

\section{- Advantages}

(a) Simple method.

(b) Easy implementation.

- Disadvantages

(a) Less accurate (Lochan et al., 2016a).

(b) Difficulty in determining spring constant (stiffness) (Theodore and Ghosal, 1995).

\subsubsection{Assumed modes method (AMM)}

In AMM, the link flexibility is represented by a combination of separable mode shapes and time-varying generalized coordinates. The modal series is truncated to a finite dimension because the contribution of higher modes to the overall movement is negligible, and dynamics of the system are dominantly governed by only the first few (low frequency) modes (Tokhi and Azad, 2008). This method is usually adopted when the global shape functions can be analytically computed, like in the case of links with simple geometries.

The proper choice of suitable boundary conditions must be made while using AMM. Rahimi and Nazemizadeh (2014) and Lochan et al. (2016a) described four main boundary conditions that are applicable according to general beam vibration theories: pinned-pinned, 
clamped-pinned, clamped-free, and clamped-clamped. Ata et al. (2012) analyzed different combinations of boundary conditions and their effect on the elastic deflection and corresponding actuators' torques in a twolink FLM. If the inertia ratio of the lightweight link to the hub is small, the clamped (clamped-mass) boundary condition yields better results than pinned boundary conditions (Barbieri and Ozguner, 1988). Clamped boundary conditions can be enforced by closing a feedback control loop around the joint (Luca and Siciliano, 1991). Compatible joint variables and deflection variables and their corresponding shape functions must be selected (Book, 1990). If the joint angle is measured from the tangent of the preceding beam link to the tangent of the following beam link, clamped-free boundary conditions are a good choice for basis shapes, and if the angle between lines that connects successive joint axes of the arm is used as joint variables, pinned-pinned boundary conditions provide the necessary constraints and are good choices (Kurfess, 2018).

Effects of payload on the FLM modeled using AMM with clamped-mass boundary conditions were discussed by Ahmad et al. (2008) and Moh. Khairudin (2008). Luca and Siciliano (1991) used different payload conditions for modal analysis in a planar twolink FLM. Celentano and Coppola (2011) proposed a computationally efficient method based on the AMM to model FLMs. Suarez et al. (2018) used AMM to model a flexible link in a long-reach manipulator with a lightweight dual-arm. Gao et al. (2018) used AMM to develop an $n$-dimensional discretized model of a twolink flexible manipulator where the dynamic behavior of the flexible link is derived from the Lagrangian and the Euler-Bernoulli beam theory. Saeed et al. (2019) and Badfar and Abdollahi (2019) modeled the dynamics of rigid-flexible manipulators using the AMM technique. Other recent works on single-link FLMs using AMM include, but are not limited to, the studies done by Ouyang et al. (2017), Reddy and Jacob (2017), Meng et al. (2018), and Zhang et al. (2019a). Furthermore, the AMM was used to model two-link FLMs by Lochan and Roy (2018), Qiu et al. (2019), and Lochan et al. (2020).

\section{- Advantages}

(a) The concept of natural frequencies is explicit.

(b) A model derived with AMM is of low order that is advantageous for a control design (Sayahkarajy et al., 2016).

- Disadvantages

(a) In the AMM, it is difficult to calculate modes of the link with varying cross-sections (Theodore and Ghosal, 1995). (b) The selection of an accurate set of boundary conditions (assumed modes) is challenging (error-prone) based on manipulator structure, payload, hub inertia, and natural modes of vibration (Korayem et al., 2012).

(c) The complexity of the model increases with the increase in the number of assumed modes (Sayahkarajy et al., 2016).

\subsubsection{Finite element method (FEM)}

In FEM, the flexible link is modeled as a combination of finite number of elements, and the displacement at any point of the continuous element is expressed in terms of finite number of displacements at the nodal points multiplied by the polynomial interpolation functions (Theodore and Ghosal, 1995). Beres et al. (1993) used Lagrange formulation, and Amirouche and Xie (1993) developed a recursive formulation using Kane's equation to obtain the dynamic model of a multi-link flexible manipulator based on FEM. Zhang and Yu (2001) modeled a spatial $4 R$ manipulator with four flexible links using FEM. Korayem et al. (2009) modeled planar two-link FLM using this method. Heidari et al. (2011) proposed a nonlinear finite element model for the dynamic modeling of FLMs undergoing large deformations and tested the proposed approach in a single-link, very flexible arm. Recently, Singla and Singh (2019) used FEM to model a two-link FLM where the elastic behavior of the link is modeled using Euler-Bernoulli Beam theory and Lagrange approach to derive equations of motion. Similarly, Sahu and Patra (2016) used FEM to model a 2-DOF serial FLM. Furthermore, recent studies using FEM to obtain the mathematical model of single-link FLMs include the works by Tahir et al. (2017), and Garcia-Perez et al. (2019).

- Advantages

(a) All the generalized coordinates are physically meaningful in FEM (Lochan et al., 2016a).

(b) In FEM, the connection is supposed to be clamp-free with a minimum of two mode shapes per link (Korayem et al., 2009).

(c) Applicable for complex cross-sectional geometries (Theodore and Ghosal, 1995).

(d) FEM can handle nonlinear and mixed boundary conditions (Korayem et al., 2009; Tokhi and Azad, 2008).

(e) Fewer mathematical operations are required for inertia matrix computation in the FEM model (Theodore and Ghosal, 1995).

- Disadvantages 
(a) The use of FEM to approximate flexibility gives rise to an over-estimated stiffness matrix, which may lead to an unstable closedloop response (Theodore and Ghosal, 1995).

(b) The FEM is computationally very expensive because of a large number of state-space equations (Theodore and Ghosal, 1995; Beres et al., 1993; Korayem et al., 2012).

(c) The concept of natural frequency is not explicit (Lochan et al., 2016a).

\subsubsection{Transfer matrix method (TMM)}

In TMM, each element of the system is represented by a transfer matrix that transfers a state vector from one end of the element to the other, and the system transfer matrix is obtained by multiplying the element transfer matrices together (Krauss and Book, 2007; De Luca and Book, 2016). Krauss and Book (2007) developed Python software modules for modeling and control design of flexible robots using the TMM. Although the TMM can be used to model the FLMs without any spatial discretization or mode shape assumptions, it leads to infinite dimensional transfer functions involving transcendental expressions in the Laplace variable $s$ and hence, it is limited to the linear system. It is impossible to compute inverse Laplace transform of the infinite dimensional transfer functions; therefore, it is difficult to perform the time domain simulations. This problem of traditional TMM is overcome by the discrete-time transfer matrix method (DT-TMM), allowing nonlinear systems to be analyzed and facilitating time-domain simulations Krauss and Okasha (2013).

$\mathrm{Li}$ and Zhang (2016) combined the discrete-time transfer matrix method with the finite segment method and proposed a computationally efficient method for the modeling of FLMs. Kivila et al. (2017) elaborated a systematic method for finding natural frequencies and mode shapes for $n$-link spatial serial manipulators using TMM and validated the method by finite element analysis and experiments. An improved approach for spatial discretization of transfer matrix models of flexible structures was proposed by Krauss (2019) for converting a TMM model to a state-space model for systems with significant actuator or zero dynamics. Apart from the capabilities of TMM in feedback control design using Bode plots, discretization approaches can be used to obtain a state-space form from a TMM model so that modern (state-space) control design techniques like pole-placement can be applied (Krauss, 2019).

- Advantages (a) This approach avoids the possibility of modal spillover because a reduced-order model is never used (Krauss, 2012).

(b) The TMM is well suited to modeling FLMs when the model is used for control design because it is a frequency domain technique that outputs Bode plots, which makes it easy to incorporate feedback (Krauss and Book, 2007).

(c) The establishment of global dynamic equations for modeling a system is not needed ( $\mathrm{Li}$ and Zhang, 2016).

(d) The orders of the matrices involved in the calculation always remain small regardless of the number of elements in the model, which significantly increases the computational speed (Li and Zhang, 2016).

- Disadvantages

(a) It makes no allowance for interaction between the gross motion and the flexible dynamics of the manipulator, nor can these effects be easily included in the model (Tokhi and Azad, 2008).

\subsection{Methods of deriving equations of motion}

There are three common methods to derive the governing equations of motion of the FLMs, which have their own merits and demerits: Newton-Euler equations, Lagrange equations, and Kane's method. Kane and Levinson (1980) presented the comparison of different methods for deriving the equations of motion. In addition to these commonly used methods, other techniques to obtain equations of motion of FLMs includes, but are not limited to, Hamilton's principle (Ding et al., 1989; Dogan and Morgül, 2010; Yang et al., 2018b; Yang and Tan, 2018; Yang et al., 2019; Cao and Liu, 2020; Meng and He, 2020) and Gibbs-Appell formulation (Korayem and Shafei, 2007).

\subsubsection{Newton-Euler formulation}

In this formalism, the dynamic equations are written separately for each body/link. Boyer and Glandais (1999) used the Newton-Euler formulation for obtaining the dynamic model of a spatial four degrees of freedom flexible manipulator. Sabatini et al. (2012) modeled multi-body dynamics of flexible space manipulator using the Newton-Euler approach. Subedi et al. (2020) used the Newton-Euler technique with LPM to derive equations of motion for a flexible cantilever beam. Bascetta et al. (2017) developed a closed-form 
model of three-dimensional flexible manipulator with links of general shape using the Newton-Euler formulation. Scaglioni et al. (2017) used the Newton-Euler method to obtain a closed-form control-oriented model of the highly flexible manipulators.

- Advantages

(a) Simple formulation because the equations of motion will always have the same form independently of the geometry, inertia, or constraints of motion of a rigid body (Malvezzi et al., 2019).

(b) Inverse dynamics is in real-time because the equations are evaluated in a numeric and recursive way.

(c) It may be considered better than the Lagrangian method for the implementation of model-based control schemes because the equations can be implemented by numerical programming methods.

- Disadvantages

(a) Workless constraint forces or torques must be determined, which is difficult when the system is composed of many bodies (Malvezzi et al., 2019).

\subsubsection{Lagrange formulation}

The Lagrange method eliminates interaction forces between adjacent links and provides a systematic method for developing the equations of motion of the entire system. In this formalism, the symbolic closed-form equations are obtained directly. Ahmad et al. (2008), Moh. Khairudin (2008), Abe (2009), Celentano and Coppola (2011), Ouyang et al. (2017), Ouyang et al. (2017), Reddy and Jacob (2017), Meng et al. (2018), Sun et al. (2018), Badfar and Abdollahi (2019), Lochan et al. (2019), Qiu et al. (2019), Qiu and Zhang (2019), Saeed et al. (2019), Pradhan and Subudhi (2020), and Boucetta et al. (2020) used the Lagrange formulation for obtaining the mathematical model of the FLMs with AMM.

Other recent studies using the Lagrange method for deriving equations of motion of the FLMs includes, but are not limited to, Singh and Rajendran (2016), Si et al. (2017), Dong et al. (2019), Mehria and Foruzantabarb (2019), Singla and Singh (2019), and Agrawal et al. (2020).

\section{- Advantages}

(a) Eliminates workless constraint forces to obtain constraint-free differential equations (Malvezzi et al., 2019). (b) It may be considered better than the NewtonEuler method for the study of dynamic properties and analysis of control schemes because the closed-form symbolic equations are obtained directly.

\section{- Disadvantages}

(a) Complicated derivatives of Lagrangians (energy functions), which often results in complex formulation (Kane and Levinson, 1980).

(b) Lengthy equations.

(c) Computationally inefficient (Li and Sankar, 1993).

\subsubsection{Kane's method}

Concepts of generalized speeds, angular velocities, and partial velocities are the key elements of Kane's method (Kane and Levinson, 1985). The equations of motion derived from Kane's method are superior (simpler form and requires a minimum of labor to derive) to Lagrange's equations for the formulation of equations governing modal coordinates (Kane and Levinson, 1980).

Buffinton (1992) used AMM and an alternative form of Kane's method to formulate the equations of motion of a two-degree-of-freedom manipulator (like Stanford Arm) with a translational flexible beam. Amirouche and Xie (1993) derived equations of motion of a twolink robot manipulator with a rigid link and a flexible link based on Kane's equation and the general matrix representation of the partial velocities and partial angular velocities. They used a recursive formulation based on FEM. Meghdari and Fahimi (2001) used Kanes method in conjunction with improved assumed modes technique to obtain first-order decoupled equations of motion of the two-link flexible manipulator in conjunction with choosing a proper congruency transformation between derivatives of generalized coordinates and generalized speeds. Zhang and Zhou (2006) used Kane's method to derive the equations of motion of the flexible robots and showed the effects of flexibility on the dynamics of the robot with two flexible links and one rigid link connected by three flexible joints. They used AMM to describe the deformation of the flexible links.

Recently, Ren et al. (2016) used Kane's method to establish the dynamic equations of motion of a twolink manipulator with two flexible joints/flexible links. Jia et al. (2017) used Kane's method to derive the coupled dynamics of the multi-link flexible system(with two flexible links and two rigid links) and a singular perturbation approach to decouple the dynamics of the 
whole system into a fast subsystem and a slow subsystem. Additionally, other relevant studies using Kane's method for modeling FLMs includes Malvezzi et al. (2019), Bian et al. (2018), and Bian and Gao (2018).

- Advantages

(a) Kane's method leads to simpler equations of motion (Kurfess, 2018).

(b) Differentiation scalar energy function is not required (Kane and Levinson, 1980).

(c) Computationally efficient (Kurfess, 2018).

(d) Eliminates constraints forces that ultimately do not contribute to the equations of motion to obtain constraint-free differential equations (Malvezzi et al., 2019; Kurfess, 2018).

(e) It can be used for solving the dynamics of robots containing closed-chains without cutting the closed-chain open (Ma Xiang-feng and Xu Xiang-rong, 1988).

(f) It allows the use of motion variables (generalized speeds) that permit the selection of these variables as not only individual timederivatives of the generalized coordinates but any convenient linear combinations of them (Kurfess, 2018).

- Disadvantages

(a) It is necessary to calculate the acceleration of the center of mass of each body (in the Lagrangian formulation, it is only necessary to calculate only the velocities of the center of mass of each body).

\subsection{Modeling FLMs undergoing small, moderate, and large deflections}

Link flexibility in FLMs can be modeled assuming either small, moderate, or large deflections. Most of the standard dynamic modeling approaches for FLM assume small deflections in general. This can be a reasonable assumption for most of the robotic applications but can be violated in some applications which require high accelerations, velocities, tip forces, and torques. Some studies consider moderate and large deformations to overcome the limitations of assuming small deflection. Luca and Siciliano (1991) used the Lagrangian approach with AMM to derive the closed-form equations of motion for multi-link planar FLM under small deflection assumption where the links are modeled as Euler-Bernoulli beams with proper clamped-mass boundary conditions. Boyer and Glandais (1999) proposed the dynamic model of FLMs derived using the Newton-Euler method premised on an exact nonlinear
Euler-Bernoulli field that can model moderate deformations or some second-order effects which appears in some limit cases as high velocities, accelerations, or tip forces and torques. Abe (2009), Heidari et al. (2011), Celentano (2016), Giorgio and Del Vescovo (2018), Esfandiar et al. (2017), and Scaglioni et al. (2017) developed the dynamic model of FLMs under the hypothesis of large link deformations.

\subsection{Partial differential equation (PDE) vs. ordinary differential equation (ODE) models}

The majority of the works done in FLMs rely on ODE models, which are derived by either LPM, AMM, or FEM. The finite dimensional ODE approximation of the PDE dynamic model is not precise enough to describe the distributed states of the FLMs, specifically because the FLMs are linear PDE models inherently (Yang et al., 2015). Conventional control techniques can be used with finite dimensional ODE models (Zhang et al., 2004). Although the ODE models are simple for control design and analysis, they may cause problems such as spillover instability, a high order of controllers, and difficulty in engineering implementation (Zhang and Liu, 2012b).

To avoid the problems associated with ODE models, the PDE dynamic models are studied, which are derived without any truncation of vibration modes and are precise enough to describe the distributed states. Commonly, Hamilton's principle is used to derive governing equations of the FLMs modeled using PDE.

The resulting PDE models are infinite dimensional avoiding spillover errors and the errors caused due to mode shape truncation and simplification of the boundary conditions (Zhang et al., 2004). In the case of the rigid-flexible manipulator, coupled ODEs-PDEs can be used to obtain the dynamic model of the system (Cao and Liu, 2017b).

However, the control design using PDE models is more challenging compared to ODE models (Zhang et al., 2005; Liu et al., 2017), and accurate simulation of a nonlinear PDE is itself a very complex problem (Dogan and Morgül, 2010).

Zhang et al. (2004) compared typical PDE and ODE models for the two-link flexible manipulators. Although, the PDE modeling and control strategies require design insights for simplifications and are theoretically involving, it is claimed that the PDE models are much more attractive from the stability and robust control design perspectives with guaranteed stability properties (Zhang et al., 2004). Zhang et al. (2005) derived a PDE model for a two-link flexible manipulator, and experimental hardware setup was used to val- 
idate the analytical PDE model. Recently, Dogan and Morgül (2010), Zhang and Liu (2012a), Zhang and Liu (2012b), Zhang and Liu (2012c), Zhang and Liu (2013), Yang et al. (2015), Jiang et al. (2017), Liu et al. (2017), Liu et al. (2018), Yang et al. (2018b), Yang and Tan (2018), Zhang et al. (2019b), Cao and Liu (2019), Yang et al. (2019), Li et al. (2020), Cao and Liu (2020), and Meng and He (2020) used PDE models for relevant studies of FLMs.

\section{Control of FLMs}

Although the FLMs have many advantages for their use in a wide range of applications, the problem of control for damping out oscillations quickly and effectively is challenging. The control complexities associated with FLMs include, but are not limited to, non-minimum phase in the system, non-collocation, and under-actuation (Lochan et al., 2016a). Moreover, some of the complexities like controller/observer spillover arise because of the choice of the dynamic model of the FLMs. If a finite dimensional representation of a distributed parameter system (i.e., FLMs) is used (based on LPM, AMM, or FEM) to design the control system, some of the unmodeled dynamics present in the dynamic model of the system cause control and observation spillover. This may lead to an unstable closed-loop system. Thus, it is necessary to consider the complexities associated with the control of the FLMs while carrying out mathematical modeling of the system. Nevertheless, a model-free robust controller can be used to avoid controller/observer spillover problem caused due to model truncation (Dwivedy and Eberhard, 2006). In addition to vibrations due to link flexibility, there are other problems such as friction, backlash, and gear non-linearities that should be addressed by the control algorithm designed for FLMs.

One of the critical steps in the controller design is the tuning of the control parameters. Global controller tuning for the whole workspace and for different loads is challenging in both model-based and model-free controllers. Moreover, model parameters may need tuning, even if model identification has been made. Different methods for tuning FLM controller gains include the Ziegler-Nichols method (Mohamed et al., 2016; Agrawal et al., 2020), LMI approach (Mohamed et al., 2016), dynamic particle swarm optimization method (Agrawal et al., 2020), self-tuning method using the artificial neural network (Njeri et al., 2019), self-tuning method based on nonlinear autoregressive moving average with exogenous-input (NARMAX) model of the FLM (Pradhan and Subudhi, 2020), soft computing based tuning method (Singh and Ohri, 2018), and selftuning method based on generalized minimum variance
(Qiu et al., 2019). Compared to the standard ZieglerNichols tuning method, the recent self-tuning methods have shown superior performance in the control of FLMs (Agrawal et al., 2020).

Main control problems that are studied in the literature for FLMs are categorized as follows (Lochan et al., 2016a):

(a) Tip position control (Sahu et al., 2019a; Singh and Rajendran, 2016)

(b) Joint position control (Pradhan and Subudhi, 2020)

(c) Tip trajectory tracking control (Lochan and Roy, 2018)

(d) Joint trajectory tracking control (Si et al., 2017; Huan and Xian, 2017; Lochan et al., 2020)

(e) Vibration control (Reddy and Jacob, 2017)

(f) Motion control (Wang et al., 2017; Zhang and Yu, 2001; Tian et al., 2002)

(g) Force control (Rigatos and Busawon, 2018; Bazaei and Moallem, 2010)

(h) Hybrid control (position and force (Matsuno and Yamamoto, 1993; Wang et al., 2020), position and vibration (Singh and Ohri, 2018), trajectory tracking and vibration (Garcia-Perez et al., 2019), and other combinations).

There are various control schemes reported in the literature, depending on the control problems. Jing et al. (2019) presented a state-of-the-art review on kinematic analysis and dynamic stable control of space flexible manipulators and pointed out existing problems and suggestions on dynamic stable control. Lochan et al. (2016a) surveyed different control schemes for joint position control, tip position control, joint trajectory tracking control, and tip trajectory tracking control problems for the two-link flexible manipulators. The control schemes reported are categorized into classical control, robust control, intelligent control, and hybrid control techniques. Alandoli et al. (2016) presented different control methods for regulating the motion and suppressing the vibration of single-link, twolink, and multi-link flexible manipulators. The review showed the effectiveness of model predictive control with/without piezoelectric actuators, a combination of the robust nonlinear and fuzzy compensator, linear quadratic regulator, and fuzzy logic controllers for the control and vibration suppression of FLMs. Sayahkarajy et al. (2016) reviewed different control techniques for FLMs, including classical linear control, optimum control, inversion-based control, command 


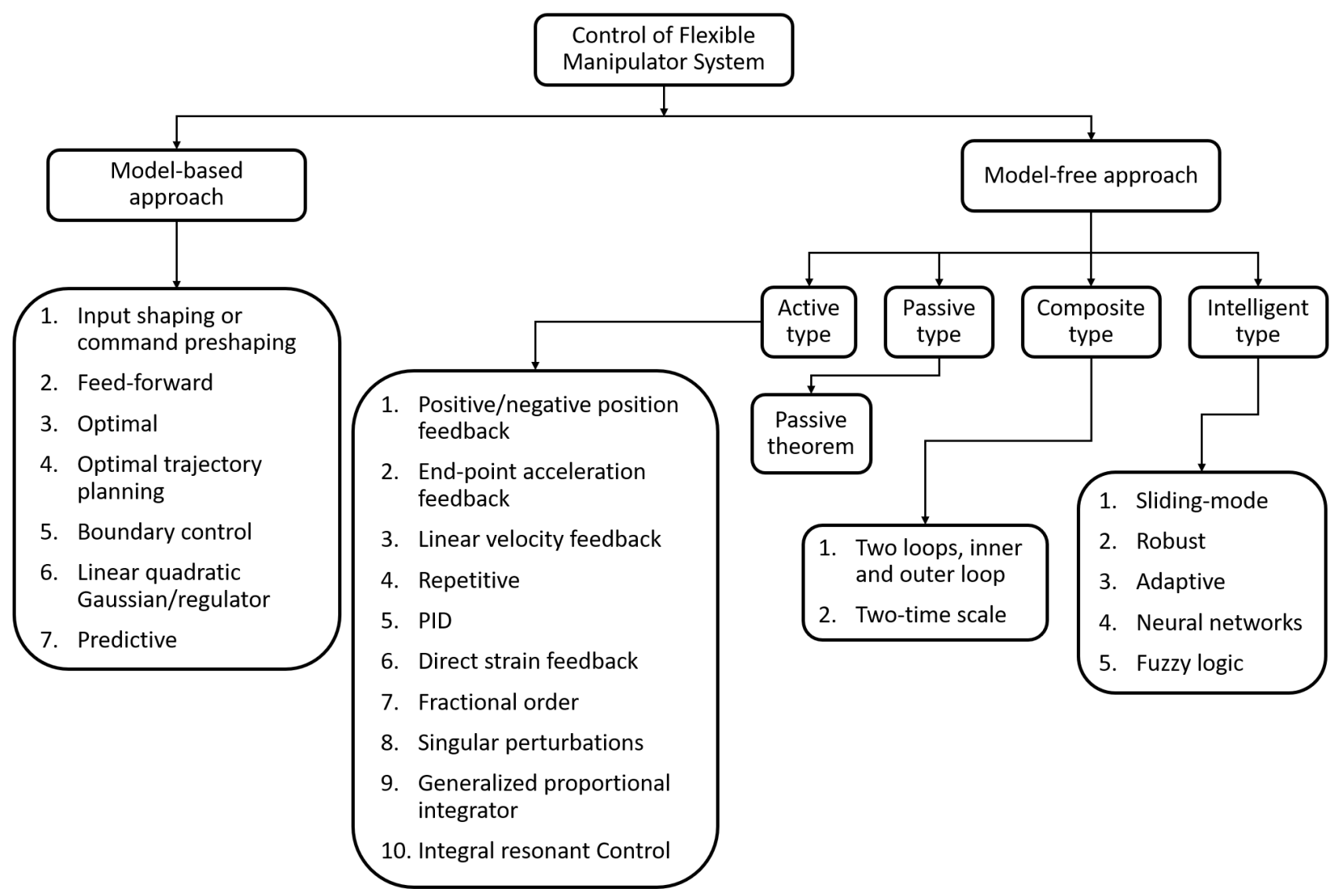

Figure 1: Control schemes

shaping techniques, trajectory optimization, force control, intelligent control techniques (using fuzzy logic, neural networks, and genetic algorithm), and robust control. Kiang et al. (2015) summarized the advantages and disadvantages associated with different control techniques used for FLMs, which are represented diagrammatically in figure 1 . Closed-loop control of the flexible manipulator system requires some forms of feedback signals to apply any suitable control strategies. The feedback control signals for the flexible manipulator system could include, but are not limited to, tip's acceleration, tip displacement, and strain along the link. Different sensors used in the control of flexible manipulators are reviewed in (Kiang et al., 2015), which include strain gauges, accelerometer, vision systems (cameras), position-sensitive devices, piezoelectric materials, ultrasonic sensor, and range sensors. Hussein (2015) reviewed different approaches of flexible robot control using visual servoing along with their advantages, disadvantages, and comparisons. Rahimi and Nazemizadeh (2014) reported a review of different intelligent control techniques of flexible manipulators by categorizing them into fuzzy logic, neural network, and genetic algorithm approaches and pointed out that intelligent control strategies, which do not need a priori knowledge of system dynamics, may provide a better way to approach control of FLMs. Benosman and Le Vey (2004) presented a survey study of different control methods used for FLMs including PD control, inputoutput linearization via static state feedback-the computed torque, adaptive control, neural network based control, lead-lag control, output redefinition, singular perturbations, sliding mode control, stable inversion in the frequency domain, stable inversion in the time domain, algebraic control, poles placement, optimal trajectory planning, optimal control, robust control, mechanical wave approach, input shaping, boundary control, and exact linearization via dynamical state feedback.

The purpose of this section is to present the review of different control schemes for the control of FLMs that are published recently (mostly from 2016 to 2019). The control techniques are divided into two main categories: model-based control and model-free control. 


\subsection{Model-based control techniques}

In model-based control, we use the system's dynamic model explicitly to aid in the controller design. The mathematical model is applied to calculate the controller response required to obtain the explicitly specified desired output response. Model-based control techniques (see figure 1) include input/command shaping control, feed-forward control, optimal control, optimal trajectory planning, boundary control, linear quadratic gaussian/regulator, predictive control, and (state) observer-based control (Kiang et al., 2015).

Since the controller is derived using the dynamic model of the system, the parameters of the model should closely match the real system to achieve good control performance. The parameters of the FLM model are obtained by best fit to measurements made during the excitation of the real robot or even a more accurate model of the robot (for example, the FEM design model). Excitation is usually made in the same frequency range as it will be used for the control of the FLM. Most of the model-based controller proposed in the literature use system identification techniques to determine precise model parameters. Then the identified parameters are used to design the controller. Krauss (2012) used an optimization algorithm to find values for the FLM system parameters that minimize the error between the experimental Bode plots and those from symbolic TMM analysis. Mohamed et al. (2016) performed system identification to obtain a set of linear models of a two-link flexible manipulator. Lou et al. (2017) proposed a reduced-order transfer function with relocated zeros and added a first-order inertia element to the model to precisely identify the system model. Ripamonti et al. (2017) identified model parameters using the Prony method and a minimization of the error between the numerical and experimental frequency response function (FRF) in the frequency domain. Cambera and Feliu-Batlle (2017) identified the parameters of the motor subsystem using the velocitytime response of the motor, and that of the flexible link subsystem using the frequency response of the system. Comi et al. (2019) and Subedi et al. (2020) used experimental measurements to identify lumped parameters of the flexible manipulators. Qiu and Zhang (2019) identified the locally controlled autoregressive moving average (CARMA) model of the flexible manipulator through experiments. Pradhan and Subudhi (2020) used a recursive least square (RLS) algorithm to identify the parameters of the nonlinear autoregressive moving average with exogenous-input (NARMAX) model of the FLM on-line. It can be noted that the model parameters of the FLM can be identified online and offline. However, the main limitation of system identification is that some of the phenomena of the original plant cannot be deduced from the input-output relationship (Njeri et al., 2018).

Input shaping is one of the traditional and effective vibration control techniques for flexible mechanical systems with known dynamic characteristics. However, it is an open-loop strategy. Therefore, the input shaping method is combined with other feedback controllers to overcome its problem of open-loop nature. Newman and Vaughan (2018) presented a concurrent design of linear feedback control with input shaping for the energy-efficient point-to-point motion of a twolink flexible manipulator while minimizing commandinduced vibration. Mehria and Foruzantabarb (2019) proposed a closed-loop input-shaping control technique based on the linear matrix inequality method to solve the problem of open-loop input shaping control. Tahir et al. (2017) designed output based filter and incorporated it with linear quadratic regulator and PID controller separately for the position and residual vibration control of single-link flexible manipulator and highlighted the superior performance of linear quadratic regulator over PID controller in tracking, vibration suppression, and robustness to payload variation. Ju et al. (2019) combined the state feedback control with the input shaping technique for vibration control of translational flexible manipulator under variable load conditions. Giorgio and Del Vescovo (2018) studied trajectory tracking and vibration control of the highly flexible planar multi-link flexible manipulator using an optimal input pre-shaping approach and feedback from the joint angles to make the system less sensitive to external disturbances.

Many classical feedback control techniques have been explored for the active vibration suppression of FLMs. Lou et al. (2017) presented experimental identification and multi-mode vibration suppression of a flexible manipulator with piezoelectric actuators and strain sensors using optimal multi-poles placement control technique. Badfar and Abdollahi (2019) used a linear matrix inequality approach to address trajectory tracking control of two-link rigid-flexible manipulator based on linear state-space model and linear quadratic regulator. Singh and Rajendran (2016) proposed an integral fast output sampling control for FLMs with the LMI approach and compared the performance of the proposed method with an observer-based discrete-time state feedback strategy.

Ghasemi (2017) proposed a filtered feedback linearization controller for the simultaneous positioning and vibration suppression of a single-link flexible manipulator that requires limited model information, specifically, knowledge of the vector relative degree, and knowledge of the dynamic-inversion matrix. Additionally, the filtered feedback linearization controller 
was combined with a high-gain adaptive law for robust control and addressed the problem of model uncertainty. Cambera and Feliu-Batlle (2017) and Cambera and Feliu-Batlle (2018) studied the input state feedback linearization controller for the tip positioning and vibration suppression of a single-link flexible link arm with and without gravity compensation, respectively. They implemented the controller into a double loop cascade control scheme, in which the inner loop is for joint position control in the presence of friction disturbances, and the outer loop is for trajectory tracking and vibration suppression of the end-effector.

Njeri et al. (2018) proposed a filtered inverse controller for the suppression of link vibration in a multilink flexible manipulator. Saeed et al. (2019) presented model-based nonlinear control of the planar two-link rigid-flexible manipulator based on the full state model of the system. Abe (2009) proposed an optimal trajectory planning technique based on particle swarm optimization algorithm for suppressing residual vibrations in two-link rigid-flexible manipulators. Zhang and Liu (2012c) proposed the optimal trajectory boundary control of the two-link flexible manipulator based on the PDE model. Cao and Liu (2018b) studied the optimal trajectory control problem for the trajectory tracking and vibration suppression of a two-link rigid-flexible manipulator based on the ODE-PDE model of the system.

Some control strategies, like input-output control, need all of the states' feedback. But, in FLMs, measuring all the flexural states is practically impossible. To overcome this problem, Mosayebi et al. (2012) proposed input-output control of FLM based on a nonlinear high gain observer to estimate the elastic degrees of freedom and their time derivatives. Reddy and Jacob (2017) proposed the Kalman filter based statedependent Riccati equation controller for accurate positioning and vibration suppression of the FLM. Ripamonti et al. (2017) presented model-based sliding mode control for vibration suppression of a three-link flexible manipulator using the linear and nonlinear sliding surface. They used a nonlinear state-space observer from the identified modal model of the FLM for sliding mode control.

Zhang and Liu (2012b) proposed observer-based boundary control for end-effector regulation, vibration suppression, and trajectory tracking of a two-link flexible manipulator in task space. The proposed method relies on the accurate dynamic model of the FLM. Liu and He (2018) presented boundary control for the onelink flexible manipulator using singular perturbation techniques that includes a position controller and a direct feedback controller to suppress the vibration. Cao and Liu (2019) proposed a controller with input quan- tization based on the PDE model of a two-link rigidflexible manipulator for joint angle control and vibration suppression.

Li et al. (2020) studied unknown control direction problem of the FLMs based on the PDE model and proposed boundary control for angle tracing and vibration elimination of the single-link flexible manipulator and adopted the Nussbaum technique in the controller design to circumvent the problem of uncertain control directions. To deal with the parametric uncertainties, varying payloads, and disturbances, adaptive and robust boundary control techniques are proposed (Liu et al., 2016; Schnelle and Eberhard, 2017). Jiang et al. (2018) proposed a boundary controller for the FLM based on the PDE robust observer to achieve the stability control, regulate the joint position, and suppress elastic vibration. He et al. (2018) designed the boundary controller with input backlash and the output feedback controller based on the PDE model to drive the FLM to the desired position, suppress its vibrations, eliminate the effects of the backlash nonlinearity and the time-varying disturbance. They further illustrated that the FLM system was better stable under the controller with output feedback. Cao and Liu (2017b) designed a boundary controller for a two-link rigid-flexible manipulator with full state constraints via Barrier Lyapunov Function.

The combination of input shaping and feedback control was used to formulate a new control approach called wave-based control, where the launch wave being a simple, shaped input, and the absorb part the feedback contribution (O'Connor, 2008). Yanan et al. (2017) proposed the wave-based control method to solve the problem of residual vibration in the pointto-point movement of a two-link flexible space manipulator. They claimed that the wave-based control has many advantages, such as not dependent on an accurate physical model, simple and easy to implement, and strong robustness. Comi et al. (2019) combined the wave-based control technique to the P/PI scheme (a cascade joint controller with an inner loop on the motor velocity and an outer loop on the load position) for vibration suppression of flexible manipulator and highlighted the superior performance of the velocity-based implementation of the wave-based controller with respect to the position-based one. Bian and Gao (2018) and Bian et al. (2018) proposed vibration control of FLMs based on internal resonance.

\section{- Advantages}

(a) Using robot dynamic models, better motion control (faster settling time and better trajectory following) can be achieved.

(b) In some cases (using feed-forward control), 
model-based control eliminates the need for the feedback sensors (e.g., force sensor), thereby reducing cost and simplifying system design.

(c) Robot paths are optimized using dynamic modeling.

(d) Enhanced robot behavior and drive performance are achieved because the paths are highly optimized by calculating the torque required to reach different positions using model-based algorithms.

(e) The mathematical model of the FLMs may be used for the model-based safety systems, for example, fast collision detection. Hence, the use of the model in more than control applications makes it advantageous from a product point of view to use model-based control methods instead of completely modelfree methods.

- Disadvantages

(a) They rely on the accurate model of the FLM system to be controlled, which is difficult and challenging to obtain. However, the model of the FLM system may be needed for design, identification, and collision detection.

(b) The control input (designed for open-loop model-based control) does not account for changes in the system, like varying payload. However, it could be possible to change parameters accurately, for example, payload and load inertia to obtain a seamless motion after gripping an object. The payload parameters are known or can be identified by the robot. This functionality is implemented in industrial robots for several years.

(c) Open-loop model-based control is not robust to parameter uncertainties and disturbances. However, it could be handled by online servos and model parameter control, which is used for industrial robots today.

(d) They require the solution of a numerical optimization problem, which can be a daunting task, especially when nonlinear models are needed that lead to non-convex problems. However, this problem is less significant if the calculations can be performed offline (Depraetere et al., 2014).

\subsection{Model-free control techniques}

Model-free control is a technique to control complex systems by using a simplified representation of the sys- tem and subsequent algebraic estimation techniques to design a simple, but effective, controller (Sira-Ramírez et al., 2017). The controller does not rely on the precise mathematical model of the system but solely based on the measurements obtained from the system. Recently, there is a trend of using the combination of different control techniques to achieve better control performances than using individual control methods. Model-free control techniques (see figure 1) include, but are not limited to, robust control, adaptive control, sliding mode control, intelligent control methods, composite control (inner/outer loop control, two-time scale control), PID control, singular perturbations technique, integral resonant control, generalized proportional integrator control, fractional order control, direct strain feedback control, repetitive control, passivity-based control, positive/negative position feedback control, end-point acceleration feedback control, and linear velocity feedback control (Kiang et al., 2015).

Mohamed et al. (2016) proposed a robust PD controller based on linear matrix inequality for joint position control of the two-link flexible manipulator under various payload conditions. They claimed that the proposed control provided better robustness and system performance compared to Ziegler-Nichols tuned PD controller. Cao and Liu (2018a) studied the actuator fault problem for a two-link rigid-flexible manipulator system and designed a novel controller that included a PD feedback structure, a disturbance observer, and a fault-tolerant algorithm for joint position regulation and vibration suppression in the presence of disturbance and actuator fault. Abd Latip et al. (2019) designed an adaptive PID controller for the joint position and tracking control of a single-link flexible manipulator, which may automatically online tune the control gains to accommodate the actuator fault. Boucetta et al. (2020) compared PD, fuzzy PD, and fractional order fuzzy PD controllers in terms of trajectory tracking, vibration suppression, robustness against disturbances, and energy consumption to control the rigidflexible two-link manipulator. They highlighted that the fractional order fuzzy PD controller performed better than other controllers. Agrawal et al. (2020) compared a modified optimal PIDD ${ }^{2}$ (proportional, integral, derivative, and second-order derivative) controller with the PID controller for controlling the position and trajectory of the single-link flexible manipulator with minimum tip oscillation. They reported the superior performance of the PIDD ${ }^{2}$ controller through simulation. Singh and Ohri (2018) presented a comparative study of different nature-inspired soft computing based PID control tuning strategies, including genetic algorithm, ant colony optimization, and particle swarm op- 
timization for the position and vibration control of a single-link flexible manipulator. Pradhan and Subudhi (2020) proposed a nonlinear self-tuning PID controller to control the joint position and link deflection of the FLM subjected to varying payloads.

Fareh et al. (2020) presented robust active disturbance rejection control for FLM to solve joint trajectories tracking control problem and minimize the link's vibrations. Xu et al. (2018) proposed a second order non-singular terminal sliding mode optimal control technique for a two-link flexible manipulator with uncertain model parameters. Lochan et al. (2016b) and Lochan and Roy (2018) used the sliding mode control technique with the PID sliding surface and the second-order sliding surface respectively, to control a two-link flexible manipulator. Yang and Tan (2018) designed a sliding mode control for joint position control and vibration suppression of a single-link flexible manipulator by using an adaptive neural approximator to compensate for the modeling uncertainties and external disturbances. Si et al. (2017) proposed a fast non-singular terminal sliding mode control for trajectory tracking of the two-link flexible manipulators with payload and external disturbances. Backstepping control based on a continuous-time extended state observer and a sampled-data extended state observer was proposed by Sahu and Patra (2016) and Sahu et al. (2019b), respectively, to solve tip tracking control problem of two-link flexible manipulator. Wang et al. (2018) proposed an adaptive fault-tolerant controller using the backstepping technique for a flexible manipulator with bounded disturbance, actuator partial failure, and output constraints.

Huan and Xian (2017) presented a backstepping control method for trajectory tracking of the two-link flexible manipulator based on extended state observer, which estimates the uncertainties as well as the system state vector. Zhao et al. (2019) studied the disturbance observer-based boundary control to achieve the vibration control, disturbance attenuation, and desired angular positioning of the FLM. Liu and Liu (2017) proposed boundary control of the FLM with input disturbances and output constraints using disturbance observer to regulate the angular position and suppress elastic vibration simultaneously. Liu et al. (2017) proposed disturbance observer-based boundary control for trajectory tracking and vibration suppression of a single-link flexible manipulator with the input saturation and external input disturbances. They used smooth hyperbolic function for handling input saturation. Liu et al. (2018) proposed boundary control with disturbance observers to solve the trajectory tracking and the vibration suppression control problems of the FLMs in $3 D$ space. Yang et al. (2018b) proposed in- finite dimensional disturbance observer-based control to solve the problems of distributed disturbances and output constraints while achieving the joint position regulation and vibration suppression.

He et al. (2017b) studied the problems of input and output constraints and designed a robust adaptive boundary controller with the disturbance observer for the control of single-link flexible manipulator with unknown system parameters and time-varying disturbances. Cao and Liu (2020) studied joint angle control and vibration suppression control of a two-link rigidflexible manipulator in $3 D$ space under input saturation using the hyperbolic tangent function.

Neural network models can be used to approximate the underlying dynamics of the FLMs to overcome the difficulty of obtaining the accurate dynamic formulation of the manipulator(Tian et al., 2002). Gao et al. (2018) investigated full-state and output feedback neural network controllers for a two-link flexible manipulator based on a radial basis function neural network to achieve trajectory tracking and vibration suppression. They claimed that the proposed adaptive neural network controller had better performance than the PD controller. Ouyang et al. (2017) proposed a reinforcement learning control to suppress the vibration of a single-link flexible manipulator by using two radial basis function neural networks: actor neural network to design proper control input and critic neural network to approximate the cost function of the system. Sun et al. (2016) used adaptive neural networks for control design using full-state feedback and output feedback separately to suppress the vibration of single-link flexible manipulator and highlighted better control performance than the PD control strategy. However, input nonlinearities were not considered in the control design. Sun et al. (2018) investigated fuzzy neural network control for trajectory tracking and vibration suppression of single-link flexible manipulator and compared the control performance of the proposed technique with the PD control. He et al. (2017a) designed the adaptive full state feedback neural network controller and the adaptive output feedback neural network controller to suppress the vibration of a single-link flexible manipulator with input deadzone. Radial basis function neural networks are used to approximate the effect of input deadzone and unknown dynamics of the FLM. Qiu and Zhang (2019) proposed a diagonal recurrent neural network control technique for the vibration control of the single-link flexible manipulator using non-contact vibration measurement based on structure light sensor. They also proposed a trajectory optimization technique to obtain optimal vibration suppression trajectory using the particle swarm optimization algorithm. Qiu et al. (2019) presented experimental studies on ac- 
tive vibration control of a two-link flexible manipulator by employing three control algorithms: generalized minimum variance self-tuning control, Takagi-Sugeno model-based fuzzy neural network control, and PD control. Furthermore, they evaluated the effectiveness of the first two controllers as compared to that of the PD control in terms of vibration suppression. Zhang et al. (2019b) designed a weighted multiple neural network boundary controller for joint position tracking and vibration suppression of a single-link flexible manipulator with uncertain parameters. Njeri et al. (2019) presented a self-tuning strain feedback gain controller for high-speed vibration control of a $3 D$ two-link flexible manipulator using the artificial neural network and highlighted that the tuned gains achieved better performance than that achieved by using fixed gain in terms of link strain and joint trajectories.

Cao and Liu (2017a) proposed an adaptive boundary iterative learning control scheme with a PD feedback structure for the joint trajectory tracking and vibration suppression of a two-link rigid-flexible manipulator with parametric uncertainties and external disturbances. Liu and Liu (2018) investigated the adaptive iterative learning control scheme for joint position tracking and vibration suppression of a single-link flexible manipulator in the presence of external disturbance and output constraints. Dong et al. (2019) proposed iterative learning control combined with an open-closedloop PD scheme for trajectory tracking of a singlelink flexible manipulator even in the presence of disturbances and uncertainties. In the proposed method, an angular correction term was introduced to achieve self-adjustment of the iterative learning control law by using angular relationships of the system output vectors. Yang et al. (2019) proposed adaptive distributed iterative learning control by combining a PD feedback structure and an iterative term for simultaneous trajectory tracking and vibration suppression of a single-link flexible manipulator subjected to system parameters uncertainties and spatio-temporal distributed disturbances. Chen et al. (2019) presented iterative learning control for simultaneous trajectory tracking and vibration suppression of a single-link flexible manipulator with uncertain parameters and unknown repetitive disturbances. Meng and He (2020) addressed the problem of the trajectory tracking control of rigid hub and vibration suppression of the flexible link simultaneously for a single-link flexible manipulator by using iterative learning control law.

Meng et al. (2018) designed a fast, stable control technique based on system energy for a planar singlelink flexible manipulator for joint position control and vibration suppression. Moreover, they proposed an online optimization method based on fuzzy-genetic algo- rithm to optimize the controller design parameter and improve the performance of the system. Zhang et al. (2019a) designed a nonlinear controller based on energy analysis for joint position control and vibration suppression control of the single-link flexible manipulator.

Yang et al. (2018a) studied the problems of tip position regulation and vibration suppression of FLM using model-free composite control based on adaptive dynamic programming. Jia et al. (2017) proposed a composite control strategy for a flexible space manipulator as a combination of an adaptive sliding mode controller for the slow subsystem and an adaptive controller for the fast subsystem. Lochan et al. (2019) designed a composite controller using contraction theory, which consists of two separate controllers designed for the slow-subsystem and fast subsystem for fast trajectory tracking and quick vibration suppression of a twolink flexible manipulator. Xu (2017) investigated the singular perturbation theory based composite learning control of FLM using neural networks and disturbance observer. Sliding mode control was used for the fast dynamics and intelligent control based on neural network, and disturbance observer was used for the slow dynamics.

\section{- Advantages}

(a) An accurate mathematical model of the system is not required, which is complex and difficult to obtain.

(b) Simpler and easier to implement than modelbased approaches.

(c) Adaptive controllers deal with the correct adjustment of the control parameters online in order to incorporate the effect of disturbances and model uncertainties (Schnelle and Eberhard, 2017).

(d) Active disturbance rejection control technique has an excellent disturbance rejection capability (Fareh et al., 2020).

\section{- Disadvantages}

(a) There is a problem of input delay in the feedback loop because the estimations of the system states are calculated based on the sensor reading.

(b) Pure sensor-based approaches will not be able to act in advance, which is potentially possible with a model-based approach and feed-forward control. 


\section{Conclusion}

This paper presented a survey of different modeling and control techniques for flexible manipulators. In recent years, the PDE models have gained increasing popularity because of the underlying advantages of infinite dimensional models that avoid spillover and model truncation errors. The review of the recent literature shows that limited research has been carried out in the modeling and control of FLMs undergoing large deflection. Because of the difficulty in obtaining the accurate dynamic model of the FLMs, model-free control methods are used more often than the model-based controllers. Moreover, model-free controllers are popular because of their robustness to parameter uncertainty and disturbances in FLMs. On the contrary, only a few model-free methods (for example, PID control and iterative learning control) are used to control industrial robots. Other model-free methods may need additional attention to solve the problem of low dynamic accuracy and/or unstable control because of the non-collocated system.

In order to solve the problem of individual controllers, there is a trend of combining two or more control techniques to achieve better control performance than using individual control techniques. A recent boost in computing power and the emergence of machine learning algorithms has enabled scientists to apply intelligent control techniques or combine learningbased control approaches with other methods to control FLMs. It is visible that a lot of recent control techniques proposed in the literature are based on neural networks and other learning-based algorithms. This literature review on the state-of-the-art modeling and control techniques of FLMs reveals that most of the studies are limited to planar single-link and twolink flexible manipulators. More research studies are required for the modeling and control of multi-link flexible manipulators undergoing general $3 D$ motion. Benchmarking and understanding the merits and limitations of the recent modeling and control methods is required to use them in the FLM (product) development. This is challenging without the development of suitable benchmark tests.

In spite of the fact that significant advancements have been made in many aspects of FLMs over the last few decades, there are many issues yet to be resolved, and simple, robust, reliable, and effective controls of FLMs still remain a challenge. Undoubtedly, further research efforts in this area would contribute significantly to the development of lightweight flexible manipulators for space research and long reach manipulators for offshore industries to perform different robotic operations safely. The mooring operation could be executed autonomously by mounting long- reach arm/s on a floating vessel/ship. Cleaning, repair, and maintenance operations in offshore wind farms and sea farms could be carried out autonomously or semiautonomously with the reduction of oscillations in the long-reach arm. Furthermore, the construction industry can utilize the long-reach manipulator for material handling and assembly tasks. Application-oriented studies on FLMs could be one of the possible future directions of research.

\section{Acknowledgments}

The work was partially funded by the Research Council of Norway through the centre SFI Offshore Mechatronics, project 237896 .

\section{References}

Aarts, R. G. and Jonker, J. B. Dynamic simulation of planar flexible link manipulators using adaptive modal integration. Multibody System Dynamics, 2002. 7(1):31-50. doi:10.1023/A:1015271000518.

Abd Latip, S. F., Rashid Husain, A., Mohamed, Z., and Mohd Basri, M. A. Adaptive PID actuator fault tolerant control of single-link flexible manipulator. Transactions of the Institute of Measurement and Control, 2019. 41(4):1019-1031. doi:10.1177/0142331218776720.

Abe, A. Trajectory planning for residual vibration suppression of a two-link rigid-flexible manipulator considering large deformation. Mechanism and Machine Theory, 2009. 44(9):1627-1639. doi:10.1016/j.mechmachtheory.2009.01.009.

Agrawal, K., Negi, R., and Singh, N. Dynamically Tuned PIDD2 Controller for Single-Link Flexible Manipulator. In Advances in VLSI, Communication, and Signal Processing. Springer Singapore, Singapore, pages 907-924, 2020.

Ahmad, M. A., Mohamed, Z., and Hambali, N. Dynamic modelling of a two-link flexible manipulator system incorporating payload. 2008 3rd IEEE Conference on Industrial Electronics and Applications, ICIEA 2008, 2008. pages 96-101. doi:10.1109/ICIEA.2008.4582487.

Alandoli, E. A., Sulaiman, M., Rashid, M. Z., Shah, H. N., and Ismail, Z. A review study on flexible link manipulators. Journal of Telecommunication, Electronic and Computer Engineering, 2016. 8(2):93-97. 
Amirouche, F. and Xie, M. An explicit matrix formulation of the dynamical equations for flexible multibody systems: A recursive approach. Computers $\&$ Structures, 1993. 46(2):311-321. doi:10.1016/00457949(93)90195-J.

Ata, A. A., Fares, W. F., and Sa'Adeh, M. Y. Dynamic analysis of a two-link flexible manipulator subject to different sets of conditions. Procedia Engineering, 2012. 41(Iris):1253-1260. doi:10.1016/j.proeng.2012.07.308.

Badfar, E. and Abdollahi, R. Trajectory tracking of rigid-flexible manipulator based on LMI optimization approach. EEA - Electrotehnica, Electronica, Automatica, 2019. 67(2):62-67.

Barbieri, E. and Ozguner, U. Unconstrained and Constrained Mode Expansions for a Flexible Slewing Link. In 1988 American Control Conference. pages 83-88, 1988. doi:10.23919/ACC.1988.4789697.

Bascetta, L., Ferretti, G., and Scaglioni, B. Closed form Newton-Euler dynamic model of flexible manipulators. Robotica, 2017. 35(5):1006-1030. doi:10.1017/S0263574715000934.

Bazaei, A. and Moallem, M. Improving force control bandwidth of flexible-link arms through output redefinition. IEEE/ASME Transactions on Mechatronics, 2010. 16(2):380-386. doi:10.1109/TMECH.2010.2046332.

Benosman, M. and Le Vey, G. Control of flexible manipulators: A survey. Robotica, 2004. 22(5):533-545. doi:10.1017/S0263574703005642.

Beres, W., Sasiadek, J. Z., and Vukovich, G. Control and dynamic analysis of multilink flexible manipulator. Proceedings - IEEE International Conference on Robotics and Automation, 1993. 3:478-483. doi:10.1109/robot.1993.292218.

Bian, Y. and Gao, Z. Nonlinear vibration control for flexible manipulator using 1: 1 internal resonance absorber. Journal of Low Frequency Noise Vibration and Active Control, 2018. 37(4):1053-1066. doi:10.1177/1461348418765951.

Bian, Y., Gao, Z., Lv, X., and Fan, M. Theoretical and experimental study on vibration control of flexible manipulator based on internal resonance. JVC/Journal of Vibration and Control, 2018. 24(15):3321-3337. doi:10.1177/1077546317704792.

Book, W. J. Modeling, design, and control of flexible manipulator arms: a tutorial review. In 29th IEEE Conference on Decision and Control. pages 500-506 vol.2, 1990. doi:10.1109/CDC.1990.203648.
Boucetta, R., Hamdi, S., and Bel Hadj Ali, S. FlexibleLink Manipulators: Dynamic Analysis and Advanced Control Strategies, pages 19-46. Springer Singapore, Singapore, 2020. doi:10.1007/978-981-15-1819-5_2.

Boyer, F. and Glandais, N. Simulation of flexible manipulators with elastic nonlinearities. In Proceedings 1999 IEEE International Conference on Robotics and Automation (Cat. No.99CH36288C), volume 1. pages 759-766 vol.1, 1999. doi:10.1109/ROBOT.1999.770066.

Buffinton, K. W. Dynamics of Elastic Manipulators With Prismatic Joints. Journal of Dynamic Systems, Measurement, and Control, 1992. 114(1):4149. doi:10.1115/1.2896506.

Cambera, J. C. and Feliu-Batlle, V. Input-state feedback linearization control of a single-link flexible robot arm moving under gravity and joint friction. Robotics and Autonomous Systems, 2017. 88:24-36. doi:10.1016/j.robot.2016.11.019.

Cambera, J. C. and Feliu-Batlle, V. Feedback Linearizing Controller for a Single Link Flexible Arm with a Passive Gravity Compensation Mechanism. IEEE International Conference on Intelligent Robots and Systems, 2018. pages 6404-6410. doi:10.1109/IROS.2018.8594409.

Cao, F. and Liu, J. An adaptive iterative learning algorithm for boundary control of a coupled ODEPDE two-link rigidflexible manipulator. Journal of the Franklin Institute, 2017a. 354(1):277-297. doi:10.1016/j.jfranklin.2016.10.013.

Cao, F. and Liu, J. Vibration control for a rigid-flexible manipulator with full state constraints via Barrier Lyapunov Function. Journal of Sound and Vibration, 2017b. 406:237-252. doi:10.1016/j.jsv.2017.05.050.

Cao, F. and Liu, J. Adaptive actuator fault compensation control for a rigid-flexible manipulator with ODEs-PDEs model. International Journal of Systems Science, 2018a. 49(8):1748-1759. doi:10.1080/00207721.2018.1479002.

Cao, F. and Liu, J. Optimal trajectory control for a two-link rigid-flexible manipulator with ODE-PDE model. Optimal Control Applications and Methods, 2018b. 39(4):1515-1529. doi:10.1002/oca.2423.

Cao, F. and Liu, J. Boundary vibration control for a two-link rigidflexible manipulator with quantized input. JVC/Journal of Vibration and Control, 2019. 25(23-24):2935-2945. doi:10.1177/1077546319873507. 
Cao, F. and Liu, J. Three-dimensional modeling and input saturation control for a two-link flexible manipulator based on infinite dimensional model. Journal of the Franklin Institute, 2020. 357(2):1026-1042. doi:10.1016/j.jfranklin.2019.10.018.

Celentano, L. Modeling of Flexible Robots with Varying Cross Section and Large Link Deformations. Journal of Dynamic Systems, Measurement and Control, Transactions of the ASME, 2016. 138(2):112. doi:10.1115/1.4032133.

Celentano, L. and Coppola, A. A computationally efficient method for modeling flexible robots based on the assumed modes method. Applied Mathematics and Computation, 2011. 218(8):4483-4493. doi:10.1016/j.amc.2011.10.029.

Chen, T., Li, M., and Shan, J. Iterative learning control of a flexible manipulator considering uncertain parameters and unknown repetitive disturbance. In 2019 American Control Conference (ACC). pages 2209-2214, 2019. doi:10.23919/ACC.2019.8815014.

Comi, F., Miguel, A. O., Cavenago, F., Ferretti, G., Magnani, G., and Rusconi, A. Modelling, validation and control of DELIAN flexible manipulator. IFAC-PapersOnLine, 2019. 52(15):364-369. doi:10.1016/j.ifacol.2019.11.702.

De Luca, A. and Book, W. J. Robots with Flexible Elements, pages 243-282. Springer International Publishing, Cham, 2016. doi:10.1007/978-3-319-32552$1 \_11$.

Depraetere, B., Liu, M., Pinte, G., Grondman, I., and Babuška, R. Comparison of model-free and modelbased methods for time optimal hit control of a badminton robot. Mechatronics, 2014. 24(8):1021-1030. doi:10.1016/j.mechatronics.2014.08.001.

Ding, X., Tarn, T. J., and Bejczy, A. K. A general dynamic model of flexible robot arms for control. In Proceedings, 1989 International Conference on Robotics and Automation. pages 1678-1683 vol.3, 1989. doi:10.1109/ROBOT.1989.100217.

Dogan, M. and Morgül, Ö. On the control of twolink flexible robot arm with nonuniform cross section. JVC/Journal of Vibration and Control, 2010. 16(5):619-646. doi:10.1177/1077546309340994.

Dong, J., He, B., Ma, M., Zhang, C., and Li, G. Open-Closed-Loop PD Iterative Learning Control Corrected With the Angular Relationship of Output Vectors for a Flexible Manipulator. IEEE Access, 2019. 7:167815-167822. doi:10.1109/ACCESS.2019.2930559.
Dwivedy, S. K. and Eberhard, P. Dynamic analysis of flexible manipulators, a literature review. Mechanism and Machine Theory, 2006. 41(7):749-777. doi:10.1016/j.mechmachtheory.2006.01.014.

Esfandiar, H., Korayem, M. H., and Haghpanahi, M. Large deformation modeling of flexible manipulators to determine allowable load. Structural Engineering and Mechanics, 2017. 62(5):619-629.

Fareh, R., Al-Shabi, M., Bettayeb, M., and Ghommam, J. Robust Active Disturbance Rejection Control for Flexible Link Manipulator. Robotica, 2020. 38(1):118-135. doi:10.1017/S026357471900050X.

Gao, H., He, W., Zhou, C., and Sun, C. Neural Network Control of a Two-Link Flexible Robotic Manipulator Using Assumed Mode Method. IEEE Transactions on Industrial Informatics, 2018. 15(2):755765. doi:10.1109/TII.2018.2818120.

Garcia-Perez, O. A., Silva-Navarro, G., and PezaSolis, J. F. Flexible-link robots with combined trajectory tracking and vibration control. $A p$ plied Mathematical Modelling, 2019. 70:285-298. doi:10.1016/j.apm.2019.01.035.

Ghasemi, A. H. Slewing and vibration control of a single-link flexible manipulator using filtered feedback linearization. Journal of Intelligent Material Systems and Structures, 2017. 28(20):2887-2895. doi:10.1177/1045389X17704067.

Giorgio, I. and Del Vescovo, D. D. Non-linear lumpedparameter modeling of planar multi-link manipulators with highly flexible arms. Robotics, 2018. 7(4):113. doi:10.3390/robotics7040060.

He, W., He, X., and Sun, C. Vibration Control of an Industrial Moving Strip in the Presence of Input Deadzone. IEEE Transactions on Industrial Electronics, 2017a. 64(6):4680-4689. doi:10.1109/TIE.2017.2674592.

He, W., He, X., Zou, M., and Li, H. PDE Model-Based Boundary Control Design for a Flexible Robotic Manipulator with Input Backlash. IEEE Transactions on Control Systems Technology, 2018. 27(2):790797. doi:10.1109/TCST.2017.2780055.

He, X., He, W., and Sun, C. Robust adaptive vibration control for an uncertain flexible Timoshenko robotic manipulator with input and output constraints. International Journal of Systems Science, 2017b. 48(13):2860-2870. doi:10.1080/00207721.2017.1360963. 
Heidari, H. R., Korayem, M. H., Haghpanahi, M., and Feliu Batlle, V. A new nonlinear finite element model for the dynamic modeling of flexible link manipulators undergoing large deflections. In 2011 IEEE International Conference on Mechatronics. pages 375380, 2011. doi:10.1109/ICMECH.2011.5971314.

Huan, G. and Xian, W. Q. Observer based tracking control of flexible manipulator. In 2017 2nd International Conference on Advanced Robotics and Mechatronics (ICARM). pages 662-666, 2017. doi:10.1109/ICARM.2017.8273241.

Hussein, M. T. A review on vision-based control of flexible manipulators. Advanced Robotics, 2015. 29(24):1575-1585. doi:10.1080/01691864.2015.1078743.

Jia, S., Jia, Y., Xu, S., and Hu, Q. Maneuver and Active Vibration Suppression of Free-Flying Space Robot. IEEE Transactions on Aerospace and Electronic Systems, 2017. 54(3):1115-1134. doi:10.1109/TAES.2017.2775780.

Jiang, T., Liu, J., and He, W. A robust observer design for a flexible manipulator based on a PDE model. Journal of Vibration and Control, 2017. 23(6):871882. doi:10.1177/1077546315587443.

Jiang, T., Liu, J., and He, W. Boundary control for a flexible manipulator with a robust state observer. JVC/Journal of Vibration and Control, 2018. 24(2):260-271. doi:10.1177/1077546316635343.

Jing, Z., Xu, Q., and Huang, J. A review on kinematic analysis and dynamic stable control of space flexible manipulators. Aerospace Systems, 2019. 2(1):1-14. doi:10.1007/s42401-018-00024-4.

Jonker, J. B. and Aarts, R. G. A Perturbation Method for Dynamic Analysis and Simulation of Flexible Manipulators. Multibody System Dynamics, 2001. 6(3):245-266. doi:10.1023/A:1012070525137.

Ju, J. Y., Li, W., Liu, Y., and Zhang, C. Masterslave integrated control for the transverse vibration of a translational flexible manipulator based on input shaping and state feedback. Shock and Vibration, 2019. 2019. doi:10.1155/2019/8419591.

Kane, T. R. and Levinson, D. A. Formulation of Equations of Motion for Complex Spacecraft. Journal of Guidance and Control, 1980. 3(2):99-112. doi: $10.2514 / 3.55956$.

Kane, T. R. and Levinson, D. A. Dynamics, theory and applications. McGraw Hill, 1985.
Khadem, S. E. and Pirmohammadi, A. A. Analytical development of dynamic equations of motion for a three-dimensional flexible link manipulator with revolute and prismatic joints. IEEE Transactions on Systems, Man, and Cybernetics, Part B: Cybernetics, 2003. 33(2):237-249. doi:10.1109/TSMCB.2003.810439.

Kiang, C. T., Spowage, A., and Yoong, C. K. Review of Control and Sensor System of Flexible Manipulator. Journal of Intelligent and Robotic Systems: Theory and Applications, 2015. 77(1):187-213. doi:10.1007/s10846-014-0071-4.

Kim, J. S. and Uchiyama, M. Vibration mechanism of constrained spatial flexible manipulators. JSME International Journal, Series C: Mechanical Systems, Machine Elements and Manufacturing, 2003. 46(1):123-128. doi:10.1299/jsmec.46.123.

Kivila, A., Book, W., and Singhose, W. Exact Modeling of n-Link Spatial Serial Structures Using Transfer Matrices. Journal of Dynamic Systems, Measurement, and Control, 2017. 139(11). doi:10.1115/1.4036555.

Korayem, M. H., Haghpanahi, M., Rahimi, H. N., and Nikoobin, A. Finite element method and optimal control theory for path planning of elastic manipulators. Studies in Computational Intelligence, 2009. 199:117-126. doi:10.1007/978-3-642-00909-9_12.

Korayem, M. H., Rahimi, H. N., and Nikoobin, A. Mathematical modeling and trajectory planning of mobile manipulators with flexible links and joints. Applied Mathematical Modelling, 2012. 36(7):32293244. doi:10.1016/j.apm.2011.10.002.

Korayem, M. H. and Shafei, A. M. Inverse dynamic equation of motion for flexible link manipulators using recursive gibbs-appell formulation. In 2007 IEEE International Conference on Robotics and Biomimetics (ROBIO). pages 2160-2165, 2007. doi:10.1109/ROBIO.2007.4522504.

Krauss, R. An Improved Approach for Spatial Discretization of Transfer Matrix Models of Flexible Structures. In 2019 American Control Conference (ACC). pages 3123-3128, 2019. doi:10.23919/ACC.2019.8814650.

Krauss, R. and Okasha, M. Discrete-time transfer matrix modeling of flexible robots under feedback control. Proceedings of the American Control Conference, 2013. pages 4104-4109. doi:10.1109/acc.2013.6580469. 
Krauss, R. W. Computationally efficient modeling of flexible robots using the transfer matrix method. JVC/Journal of Vibration and Control, 2012. 18(5):596-608. doi:10.1177/1077546311408466.

Krauss, R. W. and Book, W. J. A Python Module for Modeling and Control Design of Flexible Robots. Computing in Science Engineering, 2007. 9(3):4145. doi:10.1109/MCSE.2007.44.

Kurfess, T. R. Robotics and automation handbook. CRC press, 2018.

Li, C. . and Sankar, T. S. Systematic methods for efficient modeling and dynamics computation of flexible robot manipulators. IEEE Transactions on Systems, Man, and Cybernetics, 1993. 23(1):77-95. doi:10.1109/21.214769.

Li, H. and Zhang, X. A Method for Modeling Flexible Manipulators: Transfer Matrix Method with Finite Segments. International Journal of Computer and Information Engineering, 2016. 10(6):1086-1093.

Li, L., Cao, F., and Liu, J. Vibration control of flexible manipulator with unknown control direction. International Journal of Control, 2020. 0(0):1-13. doi:10.1080/00207179.2020.1731609.

Li, Z., Feiling, J., Ren, H., and Yu, H. A Novel TeleOperated Flexible Robot Targeted for Minimally Invasive Robotic Surgery. Engineering, 2015. 1(1):073078. doi:10.15302/J-ENG-2015011.

Liu, J. and He, W. Boundary Control for Flexible Manipulator Using Singular Perturbation. In Distributed Parameter Modeling and Boundary Control of Flexible Manipulators, pages 27-43. Springer Singapore, Singapore, 2018. doi:10.1007/978-981-108300-6_4.

Liu, Z. and Liu, J. Boundary Control of a Flexible Robotic Manipulator With Output Constraints. Asian Journal of Control, 2017. 19(1):332-345. doi:10.1002/asjc.1342.

Liu, Z. and Liu, J. Adaptive Iterative Learning Boundary Control of a Flexible Manipulator with Guaranteed Transient Performance. Asian Journal of Control, 2018. 20(3):1027-1038. doi:10.1002/asjc.1379.

Liu, Z., Liu, J., and He, W. Adaptive boundary control of a flexiblemanipulator with input saturation. International Journal of Control, 2016. 89(6):11911202. doi:10.1080/00207179.2015.1125022.

Liu, Z., Liu, J., and He, W. Partial differential equation boundary control of a flexible manipulator with input saturation. International
Journal of Systems Science, 2017. 48(1):53-62. doi:10.1080/00207721.2016.1152416.

Liu, Z., Liu, J., and He, W. Dynamic modeling and vibration control for a nonlinear 3-dimensional flexible manipulator. International Journal of Robust and Nonlinear Control, 2018. 28(13):3927-3945. doi:10.1002/rnc.4113.

Lochan, K. and Roy, B. K. Second-order SMC for tip trajectory tracking and tip deflection suppression of an AMM modelled nonlinear TLFM. International Journal of Dynamics and Control, 2018. 6(3):13101318. doi:10.1007/s40435-017-0371-1.

Lochan, K., Roy, B. K., and Subudhi, B. A review on two-link flexible manipulators. $A n$ nual Reviews in Control, 2016a. 42:346-367. doi:10.1016/j.arcontrol.2016.09.019.

Lochan, K., Roy, B. K., and Subudhi, B. SMC controlled chaotic trajectory tracking of twolink flexible manipulator with PID sliding surface. IFAC-PapersOnLine, 2016b. 49(1):219-224. doi:10.1016/j.ifacol.2016.03.056.

Lochan, K., Roy, B. K., and Subudhi, B. Use of memristive chaotic signal as a desired trajectory for a two-link flexible manipulator using contraction theory based on a composite control technique. European Physical Journal: Special Topics, 2019. 228(10):2215-2231. doi:10.1140/epjst/e2019900038-5.

Lochan, K., Singh, J. P., and Roy, B. K. Tracking control and deflection suppression of an AMM modelled TLFM using backstepping based adaptive SMC technique, volume 581. Springer Singapore, 2020. doi:10.1007/978-981-13-9419-5_4.

Lou, J., Liao, J., Wei, Y., Yang, Y., and Li, G. Experimental identification and vibration control of a piezoelectric flexible manipulator using optimal multi-poles placement control. Applied Sciences, 2017. 7(3). doi:10.3390/APP7030309.

Luca, A. D. and Siciliano, B. Closed-Form Dynamic Model of Planar Multilink Lightweight Robots. IEEE Transactions on Systems, Man and Cybernetics, 1991. 21(4):826-839. doi:10.1109/21.108300.

Ma Xiang-feng and Xu Xiang-rong. A further study on kane's equations approach of robots dynamics. In Proceedings of the 1988 IEEE International Conference on Systems, Man, and Cybernetics, volume 1. pages 107-112, 1988. doi:10.1109/ICSMC.1988.754252. 
Malvezzi, F., Orsino, R. M. M., and Coelho, T. A. H. Lagrange's, Maggi's and Kane's Equations Applied to the Dynamic Modelling of Serial Manipulator. In Proceedings of DINAME 201\%. Springer International Publishing, Cham, pages 291-304, 2019.

Matsuno, F. and Yamamoto, K. Dynamic hybrid position/force control of a flexible manipulator. In [1993] Proceedings IEEE International Conference on Robotics and Automation. pages 462-467 vol.2, 1993. doi:10.1109/ROBOT.1993.291914.

Meghdari, A. and Fahimi, F. On the First-Order Decoupling of Dynamical Equations of Motion for Elastic Multibody Systems as Applied to a Two-Link Flexible Manipulator. Multibody System Dynamics, 2001. 5(1):1-20. doi:10.1023/A:1026576603498.

Mehria, F. and Foruzantabarb, A. Control of Flexible Link Robot using a Closed Loop Input-Shaping Approach. Journal of Artificial Intelligence in Electrical Engineering, 2019. 8(29):41-52.

Meng, Q. X., Lai, X. Z., Wang, Y. W., and Wu, M. A fast stable control strategy based on system energy for a planar single-link flexible manipulator. Nonlinear Dynamics, 2018. 94(1):615-626. doi:10.1007/s11071-018-4380-1.

Meng, T. and He, W. ILC for a Flexible Single-Link Manipulator, pages 109-128. Springer Singapore, Singapore, 2020. doi:10.1007/978-981-15-2784-5_5.

Moh. Khairudin, M. K. Dynamic Modelling of a Flexible Link Manipulator Robot Using Amm. TELKOMNIKA (Telecommunication Computing Electronics and Control), 2008. 6(3):185. doi:10.12928/telkomnika.v6i3.566.

Mohamed, Z., Khairudin, M., Husain, A. R., and Subudhi, B. Linear matrix inequality-based robust proportional derivative control of a twolink flexible manipulator. JVC/Journal of $\mathrm{Vi}$ bration and Control, 2016. 22(5):1244-1256. doi:10.1177/1077546314536427.

Mosayebi, M., Ghayour, M., and Sadigh, M. J. A nonlinear high gain observer based input-output control of flexible link manipulator. Mechanics Research Communications, 2012. 45:34-41. doi:10.1016/j.mechrescom.2012.06.004.

Newman, D. and Vaughan, J. Concurrent Design of Linear Control with Input Shaping for a Two-Link Flexible Manipulator Arm. IFAC-PapersOnLine, 2018. 51(14):66-71. doi:10.1016/j.ifacol.2018.07.200.
Njeri, W., Sasaki, M., and Matsushita, K. Enhanced vibration control of a multilink flexible manipulator using filtered inverse controller. ROBOMECH Journal, 2018. 5(1):1-19. doi:10.1186/s40648-018-0125-7.

Njeri, W., Sasaki, M., and Matsushita, K. Gain Tuning for High-Speed Vibration Control of a Multilink Flexible Manipulator Using Artificial Neural Network. Journal of Vibration and Acoustics, 2019. 141(4). doi:10.1115/1.4043241.

O'Connor, W. J. Wave-based Control of Flexible Mechanical Systems, pages 25-34. Springer Berlin Heidelberg, Berlin, Heidelberg, 2008. doi:10.1007/9783-540-79142-3_3.

Ouyang, Y., He, W., and Li, X. Reinforcement learning control of a singlelink flexible robotic manipulator. IET Control Theory and Applications, 2017. 11(9):1426-1433. doi:10.1049/iet-cta.2016.1540.

Pappalardo, C. M. and Guida, D. Development of a new inertial-based vibration absorber for the active vibration control of flexible structures. Engineering Letters, 2018. 26(3):372-385.

Pradhan, S. K. and Subudhi, B. Position control of a flexible manipulator using a new nonlinear self-Tuning PID controller. IEEE/CAA Journal of Automatica Sinica, 2020. 7(1):136-149. doi:10.1109/JAS.2017.7510871.

Pucher, F., Gattringer, H., and Müller, A. Collision detection for flexible link robots using accelerometers. IFAC-PapersOnLine, 2019. 52(16):514-519. doi:10.1016/j.ifacol.2019.12.013.

Pucher, F., Gattringer, H., and Müller, A. Investigation of the Behavior of Vibration-Damped Flexible Link Robots in End-Effector Contact: Simulation and Experiment. In Multibody Dynamics 2019. Springer International Publishing, Cham, pages 139-146, 2020.

Qiu, Z.-c., Li, C., and min Zhang, X. Experimental study on active vibration control for a kind of two-link flexible manipulator. Mechanical Systems and Signal Processing, 2019. 118:623-644. doi:10.1016/j.ymssp.2018.09.001.

Qiu, Z.-c. and Zhang, W.-z. Trajectory planning and diagonal recurrent neural network vibration control of a flexible manipulator using structural light sensor. Mechanical Systems and Signal Processing, 2019. 132:563-594. doi:10.1016/j.ymssp.2019.07.014.

Rahimi, H. N. and Nazemizadeh, M. Dynamic analysis and intelligent control techniques for flexible 
manipulators: A review. Advanced Robotics, 2014. 28(2):63-76. doi:10.1080/01691864.2013.839079.

Reddy, M. P. P. and Jacob, J. Vibration control of flexible link manipulator using SDRE controller and Kalman filtering. Studies in Informatics and Control, 2017. 26(2):143-150. doi:10.24846/v26i2y201702.

Ren, S., Chu, M., and Jia, Q. Position inner loop impedance control of flexible link and flexible joint. In 2016 4th International Conference on Sensors, Mechatronics and Automation (ICSMA 2016). Atlantis Press, pages 454-459, 2016. doi:10.2991/icsma-16.2016.81.

Rigatos, G. and Busawon, K. Flexible-Link Robots, pages 271-300. Springer International Publishing, Cham, 2018. doi:10.1007/978-3-319-77851-8_5.

Ripamonti, F., Orsini, L., and Resta, F. A Nonlinear Sliding Surface in Sliding Mode Control to Reduce Vibrations of a Three-Link Flexible Manipulator. Journal of Vibration and Acoustics, 2017. 139(5). doi:10.1115/1.4036502.

Runciman, M., Darzi, A., and Mylonas, G. P. Soft Robotics in Minimally Invasive Surgery. Soft Robotics, 2019. 6(4):423-443. doi:10.1089/soro.2018.0136.

Sabatini, M., Gasbarri, P., Monti, R., and Palmerini, G. B. Vibration control of a flexible space manipulator during on orbit operations. Acta Astronautica, 2012. 73:109-121. doi:10.1016/j.actaastro.2011.11.012.

Saeed, A., Malik, F. M., Ullah, H., Akbar, Z. A., and Mazhar, N. Model-Based Control of Planar RigidFlexible Manipulator. In 2019 IEEE 7th Conference on Systems, Process and Control (ICSPC), December. IEEE, pages 122-126, 2019.

Sahu, U. K., Mishra, A., Sahu, B., Pradhan, P. P., Patra, D., and Subudhi, B. Vision-Based Tip Position Control of a Single-Link Robot Manipulator. SSRN Electronic Journal, 2019a. pages 1416-1422. doi:10.2139/ssm.3356203.

Sahu, U. K. and Patra, D. Observer based backstepping method for tip tracking control of 2-DOF Serial Flexible Link Manipulator. In 2016 IEEE Region 10 Conference (TENCON). pages 3563-3568, 2016. doi:10.1109/TENCON.2016.7848721.

Sahu, U. K., Subudhi, B., and Patra, D. Sampled-data extended state observer-based backstepping control of two-link flexible manipulator. Transactions of the Institute of Measurement and Control, 2019b. 41(13):3581-3599. doi:10.1177/0142331219832954.

Sayahkarajy, M., Mohamed, Z., and Mohd Faudzi, A. A. Review of modelling and control of flexiblelink manipulators. Proceedings of the Institution of Mechanical Engineers. Part I: Journal of Systems and Control Engineering, 2016. 230(8):861873. doi:10.1177/0959651816642099.

Scaglioni, B., Bascetta, L., Baur, M., and Ferretti, G. Closed-form control oriented model of highly flexible manipulators. Applied Mathematical Modelling, 2017. 52:174-185. doi:10.1016/j.apm.2017.07.034.

Schnelle, F. and Eberhard, P. Adaptive nonlinear model predictive control design of a flexible-link manipulator with uncertain parameters. Acta Mechanica Sinica, 2017. 33(3):529-542. doi:10.1007/s10409017-0669-4.

Si, Y., Pu, J., and Sun, L. A fast terminal sliding mode control of two-link flexible manipulators for trajectory tracking. In 2017 Chinese Automation Congress (CAC). pages 6387-6391, 2017. doi:10.1109/CAC.2017.8243928.

Singh, N. and Rajendran, S. Integral Fast Output Sampling control for Flexible Link Manipulators with LMI approach. In 2016 IEEE 1st International Conference on Power Electronics, Intelligent Control and Energy Systems (ICPEICES). pages 1-6, 2016. doi:10.1109/ICPEICES.2016.7853698.

Singh, V. K. and Ohri, J. Simultaneous control of position and vibration of flexible link manipulator by nature-inspired algorithms. In 2018 IEEE 8th Power India International Conference (PIICON). pages 16, 2018. doi:10.1109/POWERI.2018.8704403.

Singla, A. and Singh, A. Dynamic Modeling of Flexible Robotic Manipulators. In Harmony Search and Nature Inspired Optimization Algorithms. Springer Singapore, Singapore, pages 819-834, 2019.

Sira-Ramírez, H., Luviano-Juárez, A., Ramírez-Neria, M., and Zurita-Bustamante, E. W. Chapter 4 Extensions of ADRC. In Active Disturbance Rejection Control of Dynamic Systems, pages 109-172. Butterworth-Heinemann, 2017. doi:10.1016/B978-012-849868-2.00004-6.

Suarez, A., Giordano, A. M., Kondak, K., Heredia, G., and Ollero, A. Flexible link long reach manipulator with lightweight dual arm: Softcollision detection, reaction, and obstacle localization. In 2018 IEEE International Conference on Soft Robotics (RoboSoft). pages 406-411, 2018. doi:10.1109/ROBOSOFT.2018.8404953. 
Subedi, D., Tyapin, I., and Hovland, G. Modeling and Analysis of Flexible Bodies Using Lumped Parameter Method. 2020 IEEE 11th International Conference on Mechanical and Intelligent Manufacturing Technologies (ICMIMT), 2020. pages 161-166. doi:10.1109/icmimt49010.2020.9041188.

Sun, C., Gao, H., He, W., and Yu, Y. Fuzzy Neural Network Control of a Flexible Robotic Manipulator Using Assumed Mode Method. IEEE Transactions on Neural Networks and Learning Systems, 2018. 29(11):5214-5227. doi:10.1109/TNNLS.2017.2743103.

Sun, C., He, W., and Hong, J. Neural Network Control of a Flexible Robotic Manipulator Using the Lumped Spring-Mass Model. IEEE Transactions on Systems, Man, and Cybernetics: Systems, 2016. 47(8):18631874. doi:10.1109/TSMC.2016.2562506.

Tahir, N. M., Hassan, S. M., Mohamed, Z., and Ibrahim, A. G. Output based input shaping for optimal control of single link flexible manipulator. International Journal on Smart Sensing and Intelligent Systems, 2017. 10(2):367-386. doi:10.21307/ijssis2017-216.

Theodore, R. J. and Ghosal, A. Comparison of the Assumed Modes and Finite Element Models for Flexible Multilink Manipulators. The International Journal of Robotics Research, 1995. 14(2):91-111. doi:10.1177/027836499501400201.

Tian, L., Wang, J., and Mao, Z. Constrained motion control of flexible manipulators based on a dynamic neural network. Proceedings of the IEEE International Conference on Industrial Technology, 2002. 2(3):678-683. doi:10.1109/ICIT.2002.1189246.

Tokhi, M. O. and Azad, A. K. M. Flexible robot manipulators: modelling, simulation and control, volume 68. Iet, 2008.

Wang, B., Li, S., and Liu, Z. Robust Adaptive Position/Force Control for Flexible-Link with FlexibleJoint Manipulator. In Proceedings of the 11th International Conference on Modelling, Identification and Control (ICMIC2019). Springer Singapore, Singapore, pages 1215-1227, 2020.

Wang, J., Pi, Y., Hu, Y., Zhu, Z., and Zeng, L. Adaptive simultaneous motion and vibration control for a multi flexible-link mechanism with uncertain general harmonic disturbance. Journal of Sound and Vibration, 2017. 408:60-72. doi:10.1016/j.jsv.2017.07.024.
Wang, L., Zhang, D., Liu, J., Huang, H., and Shi, Q. Adaptive Fault-Tolerant Control for a Flexible Manipulator of Output-Constrained. In 2018 IEEE 8th Annual International Conference on CYBER Technology in Automation, Control, and Intelligent Systems (CYBER). pages 1048-1052, 2018.

Wanner, J. and Sawodny, O. A lumped parameter model of the boom of a mobile concrete pump. 2019 18th European Control Conference, ECC 2019, 2019. pages 2808-2813. doi:10.23919/ECC.2019.8796004.

Wei, J., Cao, D., Liu, L., and Huang, W. Global mode method for dynamic modeling of a flexiblelink flexible-joint manipulator with tip mass. $A p$ plied Mathematical Modelling, 2017. 48:787-805. doi:10.1016/j.apm.2017.02.025.

$\mathrm{Xu}$, B. Composite learning control of flexiblelink manipulator using $\mathrm{NN}$ and DOB. IEEE Transactions on Systems, Man, and Cybernetics: Systems, $2017 . \quad$ 48(11):1979-1985. doi:10.1109/TSMC.2017.2700433.

Xu, Q., Wang, W., Xia, H., Wang, Y., and Feng, Y. Second-Order Non-Singular Terminal Sliding Mode Optimal Control of Uncertain Flexible Manipulator. In 2018 IEEE 8th Annual International Conference on CYBER Technology in Automation, Control, and Intelligent Systems (CYBER). pages 13761381, 2018. doi:10.1109/CYBER.2018.8688134.

Yanan, L., Deshan, M., Houde, L., Xueqian, W., and Bin, L. Modeling and control of a two-link flexible space manipulator using the wave-based method. Proceedings of the 29th Chinese Control and Decision Conference, CCDC 2017, 2017. 0:512-519. doi:10.1109/CCDC.2017.7978148.

Yang, C., Xu, Y., Zhou, L., and Sun, Y. Model-free composite control of flexible manipulators based on adaptive dynamic programming. Complexity, 2018a. 2018. doi:10.1155/2018/9720309.

Yang, H., Liu, J., and Lan, X. Observer design for a flexible-link manipulator with PDE model. Journal of Sound and Vibration, 2015. 341:237-245. doi:10.1016/j.jsv.2014.12.033.

Yang, H. J., Liu, J. K., and He, W. Distributed disturbance-observer-based vibration control for a flexible-link manipulator with output constraints. Science China Technological Sciences, 2018b. 61(10):1528-1536. doi:10.1007/s11431-0179280-1.

Yang, H.-J. and Tan, M. Sliding Mode Control for Flexible-link Manipulators Based on Adaptive 
Neural Networks. International Journal of Automation and Computing, 2018. 15(2):239-248. doi:10.1007/s11633-018-1122-2.

Yang, Y., Liu, Z., and Ma, G. Adaptive Distributed Control of a Flexible Manipulator Using an Iterative Learning Scheme. IEEE Access, 2019. 7:145934145943. doi:10.1109/ACCESS.2019.2946018.

Zhang, C., Yang, T., Sun, N., and Zhang, J. A Simple Control Method of Single-Link Flexible Manipulators. 3rd International Symposium on Autonomous Systems, ISAS 2019, 2019a. pages 300304. doi:10.1109/ISASS.2019.8757711.

Zhang, D.-g. and Zhou, S.-f. Dynamic analysis of flexible-link and flexible-joint robots. Applied Mathematics and Mechanics, 2006. 27(5):695-704. doi:10.1007/s10483-006-0516-1.

Zhang, L. and Liu, J. Nonlinear PDE observer design for a flexible two-link manipulator. In $2012 \mathrm{Amer}$ ican Control Conference (ACC). pages 5336-5341, 2012a. doi:10.1109/ACC.2012.6314625.

Zhang, L. and Liu, J. Observer-based partial differential equation boundary control for a flexible two-link manipulator in task space. IET Control Theory Applications, 2012b. 6(13):2120-2133. doi:10.1049/ietcta.2011.0545.

Zhang, L. and Liu, J. Optimal trajectory control of flexible two-link manipulator based on PDE model. Proceedings of the IEEE Conference on Decision and Control, 2012c. pages 4406-4411. doi:10.1109/CDC.2012.6427070.
Zhang, L. and Liu, J. Adaptive boundary control for flexible two-link manipulator based on partial differential equation dynamic model. IET Control Theory and Applications, 2013. 7(1):43-51. doi:10.1049/ietcta.2011.0593.

Zhang, X., Xu, W., and Nair, S. S. Comparison of some modeling and control issues for a flexible two link manipulator. ISA Transactions, 2004. 43(4):509525. doi:10.1016/s0019-0578(07)60165-7.

Zhang, X., Xu, W., Nair, S. S., and Chellaboina, V. S. PDE Modeling and Control of a Flexible Two-Link Manipulator. IEEE Transactions on Control Systems Technology, 2005. 13(2):301-312. doi:10.1109/TCST.2004.842446.

Zhang, X. and Yu, Y. Q. Motion control of flexible robot manipulators via optimizing redundant configurations. Mechanism and Machine Theory, 2001. 36(7):883-892. doi:10.1016/S0094-114X(01)00020-9. Zhang, Y., Li, Q., Zhang, W., Liu, Y., and Xue, Z. Weighted Multiple Neural Network Boundary Control for a Flexible Manipulator With Uncertain Parameters. IEEE Access, 2019b. 7:57633-57641. doi:10.1109/ACCESS.2019.2914077.

Zhao, Z., He, X., and Ahn, C. K. Boundary Disturbance Observer-Based Control of a Vibrating SingleLink Flexible Manipulator. IEEE Transactions on Systems, Man, and Cybernetics: Systems, 2019. pages 1-9. doi:10.1109/TSMC.2019.2912900. 